\title{
c-MET inhibition enhances the response of the colorectal cancer cells to irradiation in vitro and in vivo
}

\author{
YITAO JIA ${ }^{1}$, GUANGYAO DAI ${ }^{2,3}$, JINXI WANG ${ }^{4}$, XING GAO $^{5}$, ZHAOLONG ZHAO ${ }^{2}$, ZHIHUI DUAN $^{2}$, \\ $\mathrm{BIN} \mathrm{GU}^{2}$, WEIGUANG YANG ${ }^{2}$, JIANHUA WU ${ }^{6}$, YINGCHAO JU ${ }^{6}$, MINGXIA WANG ${ }^{7}$ and ZHONGXIN $\mathrm{ZI}^{2}$ \\ ${ }^{1}$ Third Department of Oncology, Hebei General Hospital, Shijiazhuang, Hebei 050011; ${ }^{2}$ Second Department of Surgery, \\ The Fourth Hospital of Hebei Medical University, Shijiazhuang, Hebei 050035; ${ }^{3}$ Department of Surgery, \\ The First Hospital of Shijiazhuang, Shijiazhuang, Hebei 050011; ${ }^{4}$ Fourth Department of General Surgery, \\ The First Hospital of Handan, Handan, Hebei 056002; ${ }^{5}$ Second Department of Abdominal Surgery, \\ The First Affiliated Hospital of Xingtai Medical College, Xingtai, Hebei 054001; ${ }^{6}$ Experimental Animal Center; \\ ${ }^{7}$ Department of Pharmacy, The Fourth Hospital of Hebei Medical University, Shijiazhuang, Hebei 050011, P.R. China
}

Received February 11, 2015; Accepted January 5, 2016

DOI: $10.3892 / \mathrm{ol} .2016 .4303$

\begin{abstract}
The aim of the present study was to investigate the effect of hepatocyte growth factor receptor (c-MET) inhibition on the viability of colon cancer cells and xenografts exposed to irradiation using short hairpin (sh)RNA or the c-MET inhibitor PHA665752. The underlying mechanisms were also investigated. Human colorectal adenocarcinoma HT-29 cells were infected with a lentivirus expressing shRNAs against c-MET and were irradiated at 0,2,4,6 and 8 Gy. The viability of the cells was assessed by alamarBlue ${ }^{\circledR}$ assays. Mice bearing human colon carcinoma SW620 xenografts were randomly selected to receive $2.5 \%$ dimethyl sulfoxide (DMSO), $25 \mathrm{mg} / \mathrm{kg}$ PHA665752 intraperitoneally once every 2 days for 3 weeks, irradiation at $10 \mathrm{~Gy}$, or $25 \mathrm{mg} / \mathrm{kg}$ PHA665752 intraperitoneally once every 2 days for 3 weeks followed 24 h later by irradiation at $10 \mathrm{~Gy}$. The mean tumor volume (MTV) was measured. The apoptotic rate of cells was detected by terminal deoxynucleotidyl transferase-mediated dUTP nick-end labeling (TUNEL) assays, and double stranded break marker antibody $\gamma$-H2AX and hypoxia inducible factor (HIF)- $1 \alpha$ expression was examined by immunohistochemistry. alamarBlue assays revealed that c-MET downregulation by shRNA markedly accentuated the irradiation-induced reduction in the viability of HT-29 cells compared with HT-29 cells irradiated at the same doses $(\mathrm{P}<0.05)$. A combination of irradiation and PHA665752 caused an additional reduction in the MTV
\end{abstract}

Correspondence to: Professor Zhongxin Li, Second Department of Surgery, The Fourth Hospital of Hebei Medical University, 169 Tianshan Street, Shijiazhuang, Hebei 050035, P.R. China E-mail: lizhongxin99@163.com

Key words: colorectal cancer, radiation, sensitivity, c-MET inhibitor, small interfering RNA
(382.8 $\pm 42.4 \mathrm{~mm}^{3} ; \mathrm{P}<0.01$ vs. irradiation and PHA665752, $998.0 \pm 180.6$ and $844.8 \pm 190.0 \mathrm{~mm}^{3}$, respectively). TUNEL assays revealed that irradiation and PHA665752 alone caused significant apoptosis of the SW620 cells in the tumor xenografts $(\mathrm{P}<0.01$ vs. DMSO). The apoptotic index in the tumor xenografts of mice treated with a combination of irradiation and PHA665752 was significantly increased compared with mice treated with either agent alone $(\mathrm{P}<0.01)$. The combination of irradiation and PHA665752 was also associated with a marked increase in $\gamma-\mathrm{H} 2 \mathrm{AX}$ levels and a significant decrease in HIF-1 $\alpha$ expression in the xenografts $(\mathrm{P}<0.01)$. In conclusion, c-MET inhibition sensitizes colorectal cancer cells to irradiation by enhancing the formation of DNA double strand breaks and possibly alleviating tumor hypoxia.

\section{Introduction}

Worldwide, colorectal cancer (CRC) is the third most common cancer (1). Currently, the standard regimen for newly diagnosed patients with locally advanced rectal cancer (grade, cT3/T4 and $\mathrm{cN}^{+}$) is surgery in combination with neoadjuvant radiochemotherapy $(2,3)$. However, the majority of patients have mid to advanced stage CRC at the time of diagnosis. Neoadjuvant radiochemotherapy improves the survival and anus-preservation rates by shrinking tumors, decreasing the clinical stage and reducing the pathological grade (4). While patients with local CRC have a more favorable outcome, with a 5-year survival rate of $90 \%$, patients with metastatic CRC have a poor 5-year survival rate of $12 \%$, despite the good therapeutic regimens that are available, including surgical resection, adjuvant radiotherapy and chemotherapy (5).

Hepatocyte growth factor receptor (c-MET) is a tyrosine kinase located on cell membranes, and is composed of two disulfide-linked chains, an extracellular 50-kD $\alpha$-chain and a transmembrane $140-\mathrm{kD} \beta$-chain with tyrosine kinase activity (6). The ligand of c-MET is hepatocyte growth factor (HGF), also termed scatter factor. HGF is secreted by fibroblasts in the tumor stroma, which acts on c-MET in tumor cells, 
leading to the activation of downstream signaling pathways, therefore implicating c-MET in the development, progression and metastasis of cancer (7). c-MET promotes the mitosis, migration and morphogenesis of multiple cells (8), and aberrant expression of c-MET is associated with the development and progression of multiple human malignancies (9). There are two important phenotypes that appear to be more pronounced with the activation of c-MET, metastasis and drug resistance (10). High c-MET expression has been detected in CRC and has been observed to be associated with tumor invasion and lymph node and liver metastasis $(11,12)$. It has been revealed that c-MET may upregulate the expression of hypoxia-inducible factor (HIF) in tumor cells, therefore increasing the resistance of tumor cells to radiochemotherapy (13). In addition, c-MET was revealed to induce epithelial-mesenchymal transition (EMT) in tumor cells (14). Cells that develop EMT exhibit stem cell characteristics and are resistant to radiochemotherapy (6). However, little is known concerning the effect of HGF/c-MET inhibition on the sensitivity of CRC cells to radiotherapy $(15,16)$.

The present study hypothesized that concurrent c-MET inhibition may sensitize CRC cells to irradiation. The current study investigated the effect of c-MET inhibition using short hairpin (sh)RNA or the c-MET inhibitor PHA665752 on the viability of human colon carcinoma cells and xenografts exposed to irradiation.

\section{Materials and methods}

Cells lines. The human colorectal adenocarcinoma HT-29 and colon carcinoma SW620 cell lines were purchased from the Cell Bank of Type Culture Collection of Chinese Academy of Sciences (Shanghai, China). The cells were incubated at $37^{\circ} \mathrm{C}$ in a $100 \%$ atmosphere and in complete Leibovitz's L-15 medium (M\&C Gene Technology, Beijing, China) with Gibco tetracycline-free $10 \%$ fetal bovine serum (Thermo Fisher Scientific, Inc., Waltham, MA, USA), 100 units/ml penicillin and $100 \mu \mathrm{g} / \mathrm{ml}$ streptomycin (M\&C Gene Technology).

Lentiviral infections. shRNA targeting the c-MET gene (sequence, 5'-AGAATGTCATTCTACATGAGC-3'; Promega Corporation, Madison, WI, USA) and a scrambled shRNA (sequence, 5'-ATCAGAACCAGAGGCTTGGTC-3') were separately cloned into the Bam $\mathrm{HI}$ site of a TA Cloning ${ }^{\circledR}$ vector. The scrambled shRNA and TA Cloning vector were kindly donated by Dr. Demin Zhou (School of Pharmaceutical Sciences, Peking University, Beijing, China). The lentiviral vector plasmids pSD400-c-MET-shRNA and pSD400-scr-shRNA were generated by cloning the BamHI fragment of the TA Cloning vector containing appropriate shRNAs into the BamHI site of the lentiviral vector pSD400 (Dr Demin Zhou; School of Pharmaceutical Sciences, Peking University, Beijing, China). The recombinant lentiviruses were produced by co-transfecting human embryonic kidney 293T cells with pSD400-c-MET-shRNA or pSD400-scr-shRNA and the packaging vectors pMDL, pRSV and VSV-G (human embryonic kidney $293 \mathrm{~T}$ cells and packaging vectors were obtained from the School of Pharmaceutical Sciences, Peking University, Beijing, China). This was achieved using Invitrogen Lipofectamine ${ }^{\circledR} 2000$ kit (Thermo Fisher Scientific, Inc.). The HT-29 cells were then infected with the lentiviral vectors, consisting of pSD400-c-MET-shRNA expressing shRNA against c-MET or pSD400-scr-shRNA expressing scrambled shRNA at a final concentration at $10 \mu \mathrm{g} / \mathrm{ml}$, and transfected cells were selected with $0.85 \mu \mathrm{g} / \mathrm{ml}$ puromycin (M\&C Gene Technology).

Immunoblotting assays. Cellular lysates were prepared using the RIPA lysis buffer (Dakewe Biotech Co., Ltd, Beijing, China) containing a cocktail of protease inhibitors (Roche Diagnostics, Basel, Switzerland). The cellular proteins were resolved by SDS-PAGE and electrotransferred to a polyvinyl difluoride transfer membrane. The immunoblotting procedure was performed as previously described (17), using the rabbit anti-human c-MET monoclonal antibody (dilution, 1:2,000; catalog no., ab51067; Abcam, Cambridge, MA, USA) and rabbit anti-human glyceraldehyde 3-phosphate dehydrogenase (GAPDH) monoclonal antibody (dilution, 1:1,000; catalog no., ab181602; Abcam). The protein bands were visualized using Immobilon Western Chemiluminescent HRP Substrate (catalog no., WBKLS0500; EMD Millipore, Billerica, MA, USA). Densitometry was performed by calculating the optical density $\mathrm{x}$ optical region using Molecular Analyst ${ }^{\mathrm{TM}}$ (Bio-Rad Laboratories, Inc., Hercules, CA, USA) and Image J software (version 1.410; National Institutes of Health, Bethesda, MD, USA). GAPDH acted as an internal control and c-MET protein expression was described as the c-MET/GAPDH ratio.

alamarBlue $^{\circledR}$ assays. Transfected HT-29 cells were irradiated at a dose of $0,2,4,6$ or 8 Gy and cell viability was assessed using Invitrogen alamarBlue assays, according to the manufacturer's protocol (Thermo Fisher Scientific, Inc.). Subsequent to a $72 \mathrm{~h}$ incubation, the cells were stained with alamarBlue dye and the fluorescence intensities were measured at $540 \mathrm{~nm}$ using SpectraMax Gemini XS (Molecular Devices, LLC., Sunnyvale, CA, USA), and the values were calculated with the controls set at $100 \%$. The experiments were performed $\geq 3$ times independently in 6 pairs.

Mouse xenograft studies. In total, 32 4-week-old female nude mice of the Balb/c strain were purchased from the Institute of Laboratory Animal Sciences, Chinese Academy of Medical Sciences [Beijing, China; permission no. SCXK (Jing) 2005-0013]. The mice were bred in a clean environment at the Experimental Animal Center of the Fourth Hospital Affiliated to Hebei Medical University (Shijiazhuang, Hebei, China) and fed a cobalt-60-irradiated mouse diet. The experimental protocol for the present animal study was approved by the Institutional Animal Care and Use Committee. The animal experiments were conducted in accordance with the USA National Institutes of Health guidelines for the care and use of laboratory animals (18).

In total, $5 \times 10^{6} \mathrm{SW} 620$ cells in suspended in $200 \mu$ d dimethyl sulfoxide (DMSO; Tokyo Chemical Industry, Tokyo, Japan) were subcutaneously inoculated into the left costal-abdominal region of the mice. The xenograft growth was monitored and when it reached a size of $\sim 100 \mathrm{~mm}^{3}$ at 7 days post-inoculation, the mice were randomly assigned to receive various agents, as follows: $2.5 \%$ DMSO intraperitoneally once every 2 days for 3 weeks; PHA665752 (Pfizer, Inc., New York, NY, USA) at $25 \mathrm{mg} / \mathrm{kg}$ intraperitoneally once every 2 days for 3 weeks; 
irradiation at a total dose of $10 \mathrm{~Gy}$; or PHA665752 at $25 \mathrm{mg} / \mathrm{kg}$ intraperitoneally followed $24 \mathrm{~h}$ later by irradiation at a total dose of $10 \mathrm{~Gy}$. The long and short diameters of the tumor were measured using a beam caliper every 3 days and the tumor volume was calculated using the following formula: Tumor size $=\left(\pi x\right.$ long diameter $x$ short diameter $\left.{ }^{2}\right) / 6$. The nude mice were anesthetized by intraperitoneal injection with $10 \%$ chloral hydrate (Shanghai Seebio Biotechnology, Inc., Shanghai, China), sacrificed and dissected, and the tumor xenografts were collected for subsequent experiments.

Terminal deoxynucleotidyl transferase-mediated dUTP nick-end labeling (TUNEL) assays. Apoptosis of tumor cells was detected by TUNEL assay, according to the manufacturer's protocol (Roche Diagnostics), and the tissues were incubated with peroxidase-labeled mouse anti-human anti-digoxin polyclonal antibody (dilution, 1:100; catalog no., 11684817910; In Situ Cell Death Detection kit; Sigma-Aldrich, St. Louis, MO, USA). The tissue sections were observed under a fluorescence microscope (Zeiss EM 109; Carl Zeiss AG, Oberkochen, Germany) and images were captured. In total, 5 slides were selected per treatment and 10 fields of view were randomly selected. The number of apoptotic cells was counted and averaged by two experienced pathologists, who assessed all slides and were blind to the treatment administered, under an optical microscope (BX61; Olympus Corp., Tokyo, Japan) at a magnification of x 100 . The percentage of apoptotic cells [apoptotic index (AI)] was estimated using the following formula: AI $(\%)=$ (number of apoptotic cells / total cell number) x 100 .

Immunohistochemistry. Immunohistochemistry was performed using the streptavidin peroxidase method. The tumor tissues were incubated with rabbit anti-human double stranded break antibody $\mathrm{H} 2 \mathrm{AX}$ monoclonal antibody and rabbit anti-human hypoxia inducible factor (HIF)- $1 \alpha$ monoclonal antibody (Abcam) at $4^{\circ} \mathrm{C}$ overnight. The tissues were conjugated with a secondary monoclonal rabbit anti-biotin antibody (catalog no. SP-9001; dilution, 1:200; SPlink HRP Rabbit Detection (DAB) kit; Hebei Bio-High Technology Development Co., Shijiazhuang, China), and visualized with 3,3'-diaminobenzidine. H2AX is indicated by brown-yellow staining of the nuclei, while HIF-1 $\alpha$ is indicated by brown-yellow staining of the cytoplasm and membrane of cells. Image-Pro ${ }^{\circledR}$ plus image analysis software version 6.0 (Media Cybernetics, Inc., Rockville, MD, USA) was used for quantitative analysis. The integrated optical density of the positively stained cell per unit region at each field of view was calculated, and the mean density was estimated from 3 randomly selected fields of view.

Statistical analysis. All statistical analyses were performed using SPSS software version 13.0(SPSS,Inc., Chicago,IL,USA). All numerical variables were expressed as the mean \pm standard deviation, and were analyzed using one-way analysis of variance. Pairwise comparisons were calculated using Fisher's least significant difference or Student-Newman-Keuls test, and differences of proportions were tested for statistical significance with the $\chi^{2}$ test. $\mathrm{P}<0.05$ was considered to indicate a statistically significant difference.

\section{Results}

Downregulation of c-MET expression sensitizes human colon carcinoma cells to irradiation in vitro. The immunoblotting assays revealed marked suppression of the expression of c-MET upon DOX treatment (400 and 1,000 nM; Fig. 1A). The present study evaluated the effect of c-MET downregulation on irradiation-induced cytotoxicity against human colorectal adenocarcinoma HT-29 cells. alamarBlue assays demonstrated that the irradiation caused a significant dose-dependent decrease in the viability rate of HT-29 cells (2 Gy, 69.0 $7.90 \%$; 8 Gy, 37.2 $\pm 8.02 \%$; Fig. 1B). c-MET downregulation by shRNA markedly accentuated irradiation-induced reduction in the viability of HT-29 cells compared with HT-29 cells irradiated at the same doses $(\mathrm{P}<0.05)$, which indicates that c-MET downregulation sensitizes HT-29 cells to irradiation in vitro.

c-MET inhibition accentuates the suppression of tumor xenograft growth in nude mice. In mice bearing human colon carcinoma SW620 cells, irradiation markedly reduced the MTV $\left(998.0 \pm 180.6 \mathrm{~mm}^{3}\right)$ compared to DMSO $\left(2,231.1 \pm 855.6 \mathrm{~mm}^{3}\right.$; $\mathrm{P}<0.01$; Fig. 2A). In addition, PHA665752 caused a significant reduction in the MTV $\left(844.8 \pm 190.0 \mathrm{~mm}^{3}\right.$; P<0.01 vs. DMSO). The combination of irradiation and PHA665752 caused a markedly greater reduction in the MTV $\left(382.8 \pm 42.4 \mathrm{~mm}^{3}\right.$; $\mathrm{P}<0.01$ vs. irradiation or PHA665752 alone). These results demonstrate that c-MET inhibition may accentuate the suppression by irradiation of tumor xenograft growth in vivo.

c-MET inhibition enhances the apoptosis of human colon carcinoma cells in the tumor xenograft by promoting irradiation-induced formation of DNA double strand breaks. TUNEL assays demonstrated that irradiation and PHA665752 alone caused significant apoptosis of SW620 cells in the tumor xenograft (irradiation, 18.14\%; PHA665752, 22.90\%; $\mathrm{P}<0.01$ vs. DMSO; Fig. 2B-E). The AI in the tumor xenograft of mice treated with a combination of irradiation and PHA665752 was significantly higher (36.43\%) compared with mice treated with either agent alone $(\mathrm{P}<0.01$; Fig. $2 \mathrm{~F})$.

The present study examined the expression of $\gamma-\mathrm{H} 2 \mathrm{AX}$ in SW620 cells. Irradiation of the tumor xenografts bearing SW620 cells caused a marked increase in the expression of $\gamma$-H2AX (P<0.01 vs. DMSO; Fig. 3A, B and E). PHA665752 also caused a clear increase in the expression of $\gamma-\mathrm{H} 2 \mathrm{AX}$ ( $\mathrm{P}<0.01$ vs. DMSO; Fig. $3 \mathrm{C}$ and $\mathrm{E})$. The combination of irradiation and PHA665752 leads to the greatest increase in the expression of $\gamma-\mathrm{H} 2 \mathrm{AX}(\mathrm{P}<0.01$ vs. irradiation or PHA665752; Fig. 3D and E). The present findings reveal that concurrent targeted inhibition of c-MET aggravates irradiation-induced formation of DNA double strand breaks in mouse tumor xenografts.

c-MET inhibition suppresses the expression of HIF-l $\alpha$ in mouse tumor xenografts. Immunohistochemistry revealed that irradiation and PHA665752 alone significantly suppressed the expression of HIF-1 $\alpha$ in the tumor xenografts $(\mathrm{P}<0.01$ vs. DMSO; Fig. 4A-C and E). The combination of irradiation and PHA665752 caused a significantly greater inhibition of HIF- $1 \alpha$ expression than either agent alone $(\mathrm{P}<0.01$ vs. irradiation or PHA665752; Fig. 4D and E). The 


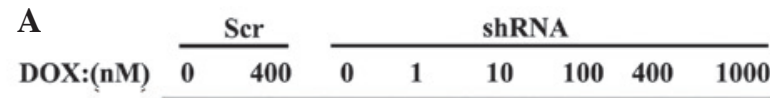

c-Met

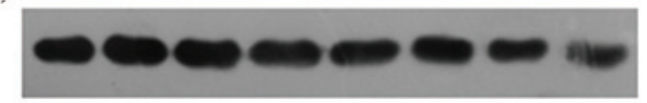

GAPDH
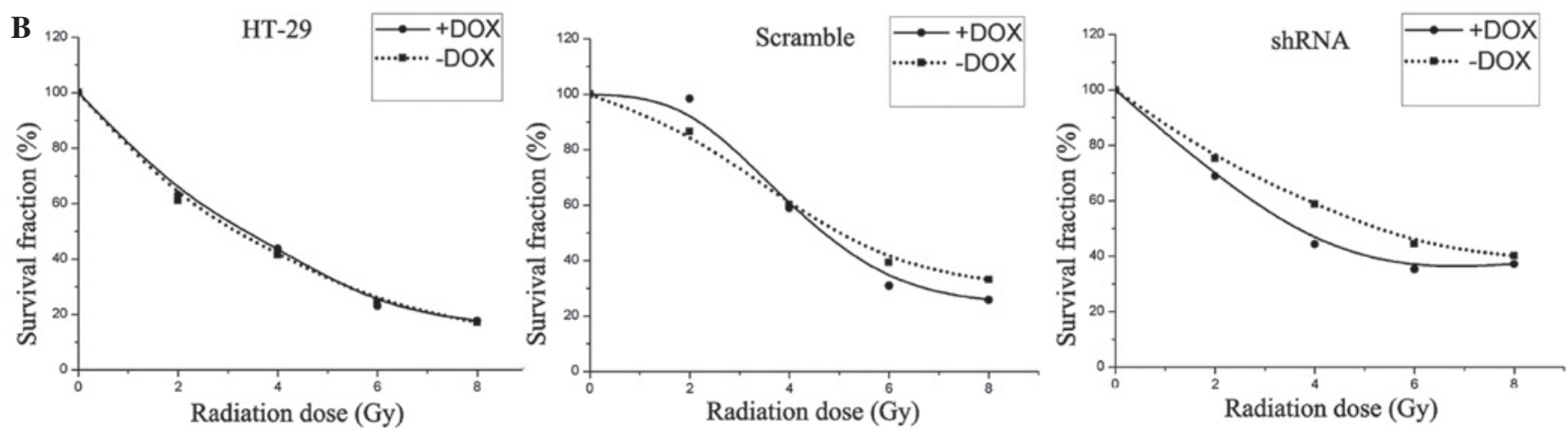

Figure 1. Human colorectal adenocarcinoma HT-29 cells were transfected with the lentiviral vectors pSD400-c-MET-shRNA or pSD400-scr-shRNA inducibly expressing shRNA against c-MET or scr, respectively. Transfected cells were treated with DOX at the indicated doses for induction of shRNA expression. (A) Immunoblotting assays were performed to examine c-MET expression. The results are representative of at least 3 independent experiments. (B) Transfected cells were irradiated at the indicated doses and cell viability was examined by Alamar Blue assays. The data are expressed as the mean \pm standard deviation of $\geq 3$ independent experiments. c-MET, hepatocyte growth factor receptor; GAPDH, glyceraldehyde 3-phosphate dehydrogenase; DOX, doxycycline; shRNA, short hairpin RNA; scr, scrambled shRNA.
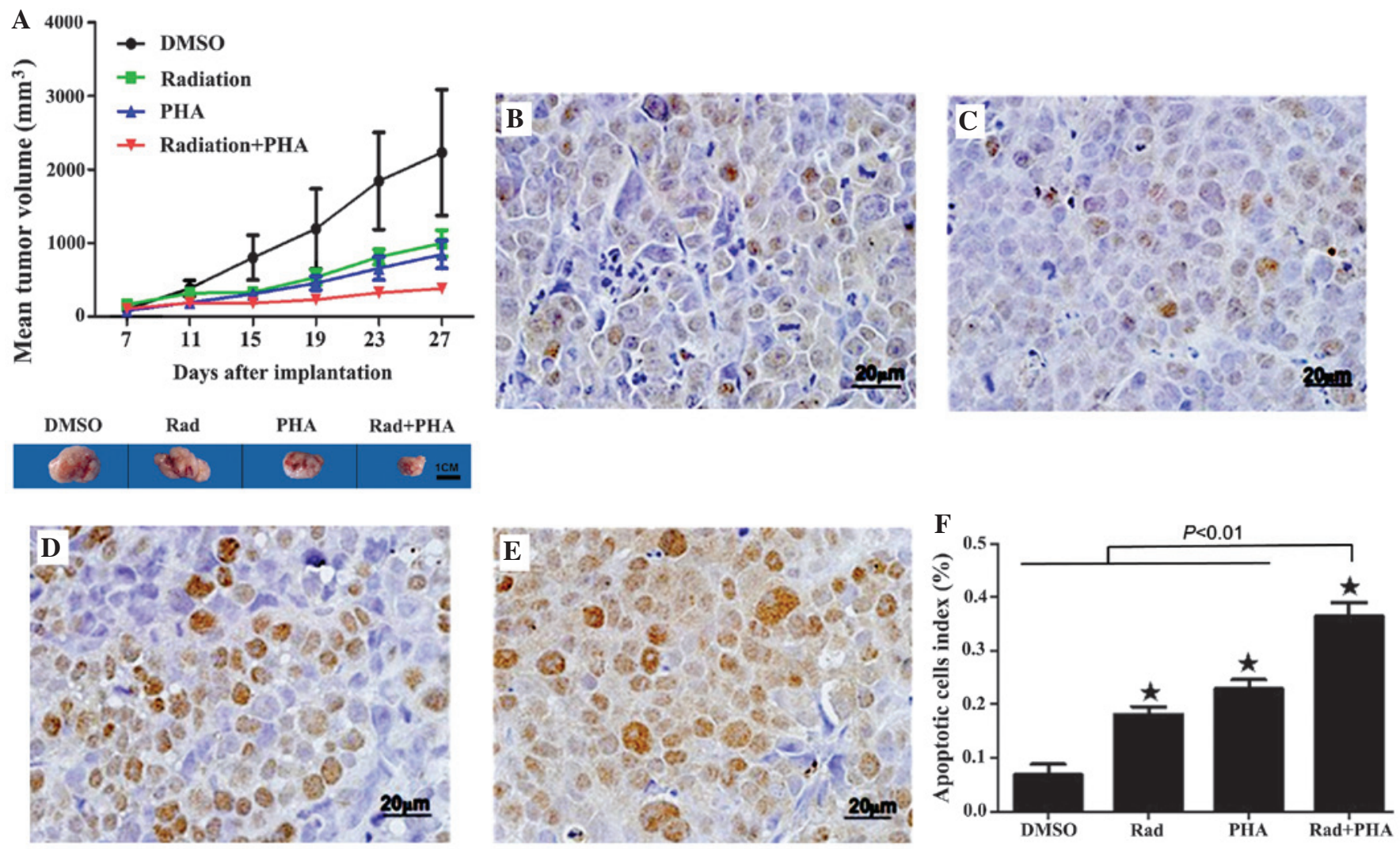

Figure 2. (A) Mice bearing human colon carcinoma SW620 xenografts were treated with 2.5\% DMSO, PHA665752, irradiation or the combination of irradiation and PHA665752. Tumor growth was monitored by measuring the mean tumor volume. $\mathrm{P}<0.01$, PHA665752, irradiation or a combination of irradiation and PHA665752 vs. DMSO; P<0.01, the combination of irradiation and PHA665752 vs. irradiation or PHA665752. (B-E) Mice were treated as in (A) and the apoptotic rate of the xenograft tissue was examined by terminal deoxynucleotidyl transferase-mediated dUTP nick-end labeling assay: (B) The DMSO group; (C) the irradiation group; (D) the PHA665752 group; and (E) the combination of irradiation and PHA665752 group. (F) The percentage of apoptotic cells in the tumor xenografts. "P<0.01, the combination of irradiation and PHA665752 vs. DMSO, irradiation or PHA665752. DMSO; dimethyl sulfoxide; PHA665752, hepatocyte growth factor receptor inhibitor; PHA, PHA665752; Rad, irradiation. 

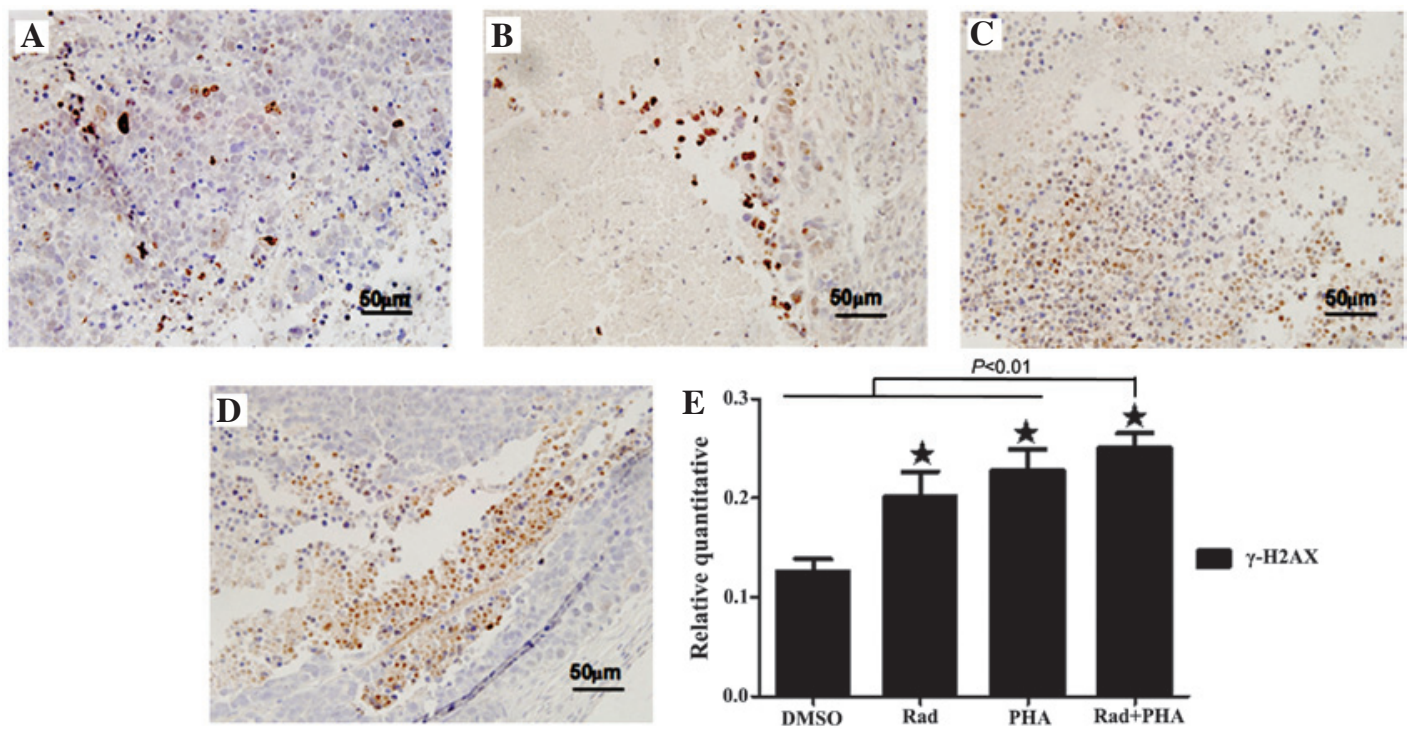

Figure 3. Mice bearing human colon carcinoma SW620 xenografts were treated with 2.5\% DMSO, PHA665752, irradiation or the combination of irradiation and PHA665752. $\gamma$-H2AX expression in the tumor xenograft was examined using immunohistochemistry in the (A) DMSO, (B) irradiation, (C) PHA665752, and (D) combination of irradiation and PHA665752 groups. (E) Quantification of $\gamma$-H2AX expression. "P $<0.01$, combination of irradiation and PHA665752 vs. DMSO, irradiation or PHA665752. $\gamma$-H2AX, a double stranded break marker; DMSO; dimethyl sulfoxide; PHA665752, hepatocyte growth factor receptor inhibitor; Rad, irradiation; PHA, PHA665752.
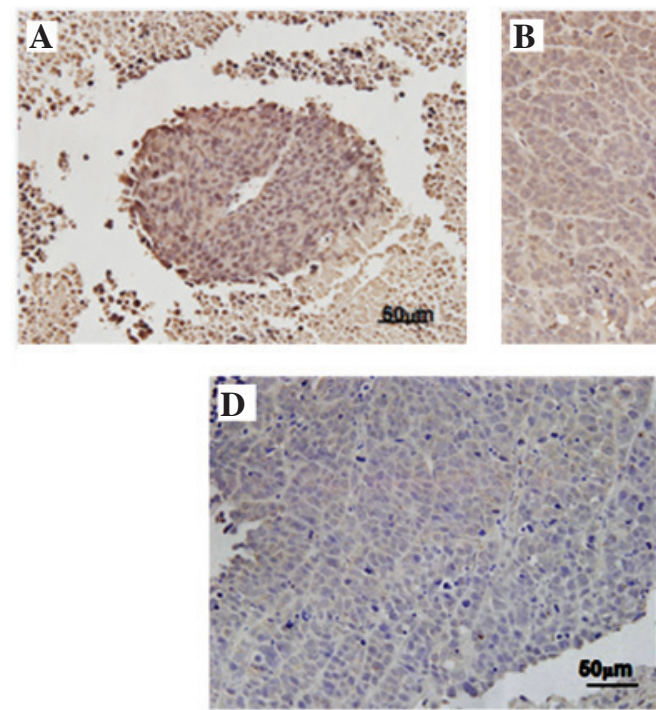
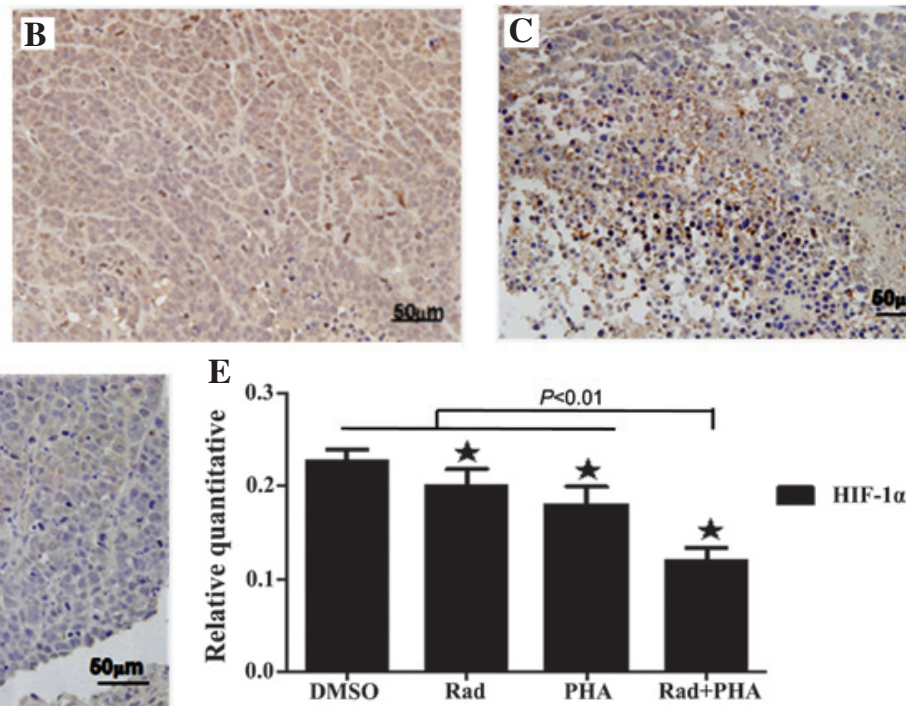

Figure 4. Mice bearing human colon carcinoma SW620 xenografts were treated with 2.5\% DMSO, PHA665752, irradiation or the combination of irradiation and PHA665752. HIF-1 $\alpha$ expression in the tumor xenograft was examined using immunohistochemistry: (A) The DMSO group; (B) the irradiation group; (C) the PHA665752 group; and (D) the combination of irradiation and PHA665752 group. (E) Quantification of HIF-1 $\alpha$ expression. "P<0.01, combination of irradiation and PHA665752 vs. DMSO, irradiation or PHA665752. HIF-1 $\alpha$, hypoxia inducible factor; DMSO; dimethyl sulfoxide; PHA665752, hepatocyte growth factor receptor inhibitor; Rad, irradiation; PHA, PHA665752.

results demonstrate that treatment with a c-MET inhibitor alleviates hypoxia in tumor tissues.

\section{Discussion}

CRC is a common malignant tumor of the gastrointestinal tract (19). This type of cancer often reaches mid to advanced stages prior to diagnosis (20). Recently, there has been a shift in the paradigm for the treatment of CRC between conventional chemotherapy and combination therapy, consisting of chemotherapy and radiotherapy, and targeted therapy, including antibodies against vascular endothelial growth factor (VEGF), such as bevacizumab, and antibodies against epidermal growth factor receptor, such as cetuximab and panitumumab $(11,21)$. However, the outcome of patients with advanced-stage CRC remains poor, therefore mandating the search for alternative effective therapies $(11,21)$. The present study hypothesized that a blockade of MET signaling may aid in overcoming resistance to radiotherapy. The present study demonstrated that c-MET downregulation by shRNA or treatment with the small-molecule c-MET inhibitor PHA665752 accentuated the cytotoxicities of irradiation against colon cancer cells in vitro and in vivo. This suggests that c-MET inhibition may require investigation as a future approach to the treatment of CRC. 
Aberrant expression of c-MET is associated with the development and progression of multiple human malignancies $(8,9)$ and has been identified as a novel target for the treatment of multiple malignant tumors $(11,22)$. Radiation has been observed to upregulate c-MET expression in a HGF-dependent or independent manner, and promotes the transcription of HGF and c-MET, while a high c-MET expression induces tumor metastasis and prevents the apoptosis of cells, resulting in radiochemotherapeutic resistance (15). In rectal cancer patients undergoing concurrent chemoradiotherapy, high expression of metastasis-associated in colon cancer-1 and c-MET was revealed to be associated with a reduced relapse-free survival rate and an adverse prognosis (23). Therefore, targeting c-MET may exhibit a synergistic effect with chemotherapy or radiotherapy $(24,25)$. The present findings are consistent with previous studies (26-29). It has been previously reported that the combination therapy of the anti-HGF monoclonal antibody AMG102 with the cytotoxic agent temozolomide or docetaxel enhances the anti-tumor action of temozolomide and docetaxel against glioblastoma multiforme (30).

Currently, the mechanisms underlying radiotherapeutic resistance in tumors mainly involve a reduction in DNA repair capacity, hypoxia and tumor cell EMT. The present study identified that PHA665752 significantly accentuated the irradiation-induced elevation of $\gamma$-H2AX expression in human colon carcinoma SW620 cells that led to enhanced apoptosis of the tumor cells, which suggests that targeted inhibition of c-MET may impair DNA repair in tumor cells caused by irradiation. It has been demonstrated that the activation of c-MET is involved in resistance to DNA damage, including radiation-induced DNA damage (31), and HGF/c-MET has been revealed to protect DNA from damage via the phosphoinositide 3-kinase/protein kinase B and proto-oncogenes RAS and RAF/mitogen-activated protein kinase pathways (32).

In addition, the present findings demonstrated that PHA665752 inhibited HIF-1 $\alpha$ expression in mice bearing SW620 xenografts, which suggests that c-MET inhibition may alleviate hypoxia in the tumor. Angiogenesis drives tumor growth (33). In addition to VEGF and the VEGF receptor (VEGFR), the HGF/c-MET signaling pathway is involved in tumor angiogenesis by upregulating the expression of pro-angiogenic factors, including VEGF and VEGFR, to promote the proliferation and migration of vascular endothelial cells, and by downregulating the expression of the angiogenesis inhibitor thrombospondin-1 (34). It has been demonstrated that anti-angiogenic treatments may normalize tumor vessels, which accelerates blood circulation and improves hypoxia in the tumor, and facilitates the aggregation of chemotherapeutic agents to the tumor microenvironment; therefore increasing the sensitivity of the tumor to radiochemotherapy $(13,35)$. To the best of our knowledge, there have been no previous studies concerning the inhibition of HIF-1 $\alpha$ expression in tumors by PHA665752.

In summary, c-MET inhibition sensitizes CRC cells to irradiation and may offer a promising approach for the treatment of locally advanced CRC.

\section{Acknowledgements}

The present study was supported by the National Natural Science Foundation of China (grant no. 81172332).

\section{References}

1. Robbins AS, Siegel RL and Jemal A: Racial disparities in stage-specific colorectal cancer mortality rates from 1985 to 2008. J Clin Oncol 30: 401-405, 2012.

2. Bosset JF, Collette L, Calais G, Mineur L, Maingon P, Radosevic-Jelic L, Daban A, Bardet E, Beny A and Ollier JC; EORTC Radiotherapy Group Trial 22921: Chemotherapy with preoperative radiotherapy in rectal cancer. N Engl J Med 355: 1114-1123, 2006

3. Gérard JP, Conroy T, Bonnetain F, Bouché O, Chapet O, Closon-Dejardin MT, Untereiner M, Leduc B, Francois E, Maurel J, et al: Preoperative radiotherapy with or without concurrent fluorouracil and leucovorin in T3-4 rectal cancers: Results of FFCD 9203. J Clin Oncol 24: 4620-4625, 2006.

4. Dhadda AS, Dickinson P,Zaitoun AM, Gandhi N and Bessell EM: Prognostic importance of Mandard tumour regression grade following pre-operative chemo/radiotherapy for locally advanced rectal cancer. Eur J Cancer 47: 1138-1145, 2011.

5. Hu T, Yao Y, Yu S, Guo H, Han L, Wang W, Tian T, Hao Y, Liu Z, Nan K and Wang S: Clinicopathologic significance of CXCR4 and Nrf2 in colorectal cancer. J Biomed Res 27: 283-290, 2013.

6. Scarpino S, Cancellario d'Alena F, Di Napoli A, Pasquini A, Marzullo A and Ruco LP: Increased expression of Met protein is associated with up-regulation of hypoxia inducible factor-1 (HIF-1) in tumour cells in papillary carcinoma of the thyroid. J Pathol 202: 352-358, 2004.

7. De Wever O, Nguyen QD, Van Hoorde L, Bracke M, Bruyneel E, Gespach $\mathrm{C}$ and Mareel M: Tenascin-C and SF/HGF produced by myofibroblasts in vitro provide convergent pro-invasive signals to human colon cancer cells through RhoA and Rac. FASEB J 18: 1016-1018, 2004

8. Park MK, Kim DK and Lee HJ: Adenoviral mediated hepatocyte growth factor gene attenuates hyperglycemia and beta cell destruction in overt diabetic mice. Exp Mol Med 35: 494-500, 2003.

9. Comoglio PM and Trusolino L: Invasive growth: From development to metastasis. J Clin Invest 109: 857-862, 2002.

10. Stellrecht CM and Gandhi V: MET receptor tyrosine kinase as a therapeutic anticancer target. Cancer Lett 280: 1-14, 2009.

11. Kammula US, Kuntz EJ, Francone TD, Zeng Z, Shia J, Landmann RG,Paty PB and Weiser MR: Molecular co-expression of the c-Met oncogene and hepatocyte growth factor in primary colon cancer predicts tumor stage and clinical outcome. Cancer Lett 248: 219-228, 2007.

12. Kataoka H, Hamasuna R, Itoh H, Kitamura N and Koono M: Activation of hepatocyte growth factor/scatter factor in colorectal carcinoma. Cancer Res 60: 6148-6159, 2000.

13. Du Z, Qin R, Wei C, Wang M, Shi C, Tian R and Peng C: Pancreatic cancer cells resistant to chemoradiotherapy rich in "stem-cell-like" tumor cells. Dig Dis Sci 56: 741-750, 2011.

14. Suárez-Causado A, Caballero-Díaz D, Bertrán E, Roncero C, Addante A, García-Álvaro M, Fernández M, Herrera B, Porras A, Fabregat I and Sánchez A: HGF/c-Met signaling promotes liver progenitor cell migration and invasion by an epithelial-mesenchymal transition-independent, phosphatidyl inositol-3 kinase-dependent pathway in an in vitro model. Biochim Biophys Acta 1853:2453-2463, 2015.

15. De Bacco F, Luraghi P, Medico E, Reato G, Girolami F, Perera T, Gabriele P, Comoglio PM and Boccaccio C: Induction of MET by ionizing radiation and its role in radioresistance and invasive growth of cancer. J Natl Cancer Inst 103: 645-661, 2011.

16. Buchanan IM, Scott T, Tandle AT, Burgan WE, Burgess TL, Tofilon PJ and Camphausen K: Radiosensitization of glioma cells by modulation of Met signalling with the hepatocyte growth factor neutralizing antibody, AMG102. J Cell Mol Med 15: 1999-2006, 2011.

17. Sun W, Song L, Ai T, Zhang Y, Gao Y and Cui J: Prognostic value of MET, cyclin D1 and MET gene copy number in non-small cell lung cancer. J Biomed Res 27: 220-230, 2013.

18. National Research Council of the National Academies: Guide for the care and use of laboratory animals. 8th edition. The National Academies Press, Washington, D.C., USA. pp199-200, 2011.

19. Jemal A, Bray F, Center MM, Ferlay J, Ward E and Forman D: Global cancer statistics. CA Cancer J Clin 61: 69-90, 2011.

20. Singh H, Daci K, Petersen LA, Collins C, Petersen NJ, Shethia A and El-Serag HB: Missed opportunities to initiate endoscopic evaluation for colorectal cancer diagnosis. Am J Gastroenterol 104: 2543-2554, 2009. 
21. Bekaii-Saab T and Wu C: Seeing the forest through the trees: A systematic review of the safety and efficacy of combination chemotherapies used in the treatment of metastatic colorectal cancer. Crit Rev Oncol Hematol 91: 9-34, 2014.

22. Teicher BA: Antiangiogenic agents and targets: A perspective. Biochem Pharmacol 81: 6-12, 2011.

23. Kawamura M, Saigusa S, Toiyama Y, Tanaka K, Okugawa Y, Hiro J, Uchida K, Mohri Y, Inoue Y and Kusunoki M: Correlation of MACC1 and MET expression in rectal cancer after neoadjuvant chemoradiotherapy. Anticancer Res 32: 1527-1531, 2012.

24. Hong TS, Wo JY and Kwak EL: Targeted therapies with chemoradiation in esophageal cancer: Development and future directions. Semin Radiat Oncol 23: 31-37, 2013.

25. Akervall J, Nandalur S, Zhang J, Qian CN, Goldstein N, Gyllerup P, Gardinger Y, Alm J, Lorenc K, Nilsson K, et al: A novel panel of biomarkers predicts radioresistance in patients with squamous cell carcinoma of the head and neck. Eur J Cancer 50: 570-581, 2014.

26. Medová M, Aebersold DM, Blank-Liss W, Streit B, Medo M, Aebi S and Zimmer Y: MET inhibition results in DNA breaks and synergistically sensitizes tumor cells to DNA-damaging agents potentially by breaching a damage-induced checkpoint arrest. Genes Cancer 1: 1053-1062, 2010.

27. Medová M, Aebersold DM and Zimmer Y: MET inhibition in tumor cells by PHA665752 impairs homologous recombination repair of DNA double strand breaks. Int J Cancer 130: 728-734, 2012.

28. Zhuang HQ, Bo QF, Yuan ZY, Wang J, Zhao LJ and Wang P: The different radiosensitivity when combining erlotinib with radiation at different administration schedules might be related to activity variations in c-MET-PI3K-AKT signal transduction. Onco Targets Ther 6: 603-608, 2013.
29. Welsh JW, Mahadevan D, Ellsworth R, Cooke L, Bearss D and Stea B: The c-Met receptor tyrosine kinase inhibitor MP470 radiosensitizes glioblastoma cells. Radiat Oncol 4: 69, 2009.

30. Jun HT, Sun J, Rex K, Radinsky R, Kendall R, Coxon A and Burgess TL: AMG 102, a fully human anti-hepatocyte growth factor/scatter factor neutralizing antibody, enhances the efficacy of temozolomide or docetaxel in U-87 MG cells and xenografts. Clin Cancer Res 13: 6735-6742, 2007.

31. Fan S, Wang JA, Yuan RQ, Rockwell S, Andres J, Zlatapolskiy A, Goldberg ID and Rosen EM: Scatter factor protects epithelial and carcinoma cells against apoptosis induced by DNA-damaging agents. Oncogene 17: 131-141, 1998.

32. Meyn RE, Munshi A, Haymach JV, Milas L and Ang KK: Receptor signaling as a regulatory mechanism of DNA repair. Radiother Oncol 92: 316-322, 2009.

33. Abdollahi A and Folkman J. Evading tumor evasion: Current concepts and perspectives of anti-angiogenic cancer therapy. Drug Resist Updat 13: 16-28, 2010.

34. Puri N, Khramtsov A, Ahmed S, Nallasura V, Hetzel JT, Jagadeeswaran R, Karczmar G and Salgia R: A selective small molecule inhibitor of c-Met, PHA665752, inhibits tumorigenicity and angiogenesis in mouse lung cancer xenografts. Cancer Res 67: 3529-3534, 2007.

35. Citrin D, Ménard C and Camphausen K: Combining radiotherapy and angiogenesis inhibitors: Clinical trial design. Int $\mathrm{J}$ Radiat Oncol Biol Phys 64: 15-25, 2006. 